Historic, Archive Document

Do not assume content reflects current scientific knowledge, policies, or practices. 



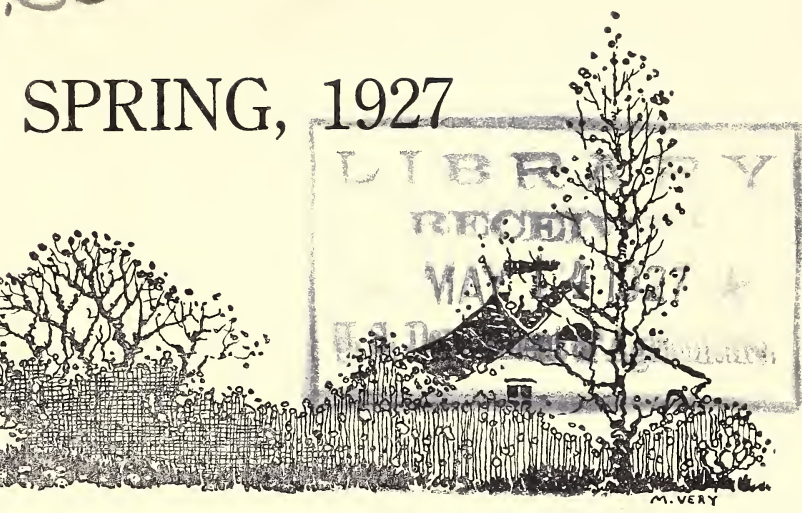

\title{
A LIST OF
}

\section{Perennials}

Annuals

Hardy Climbing Plants

IN STOCK IN OUR OWN NURSERY

\author{
\& \& \\ GARDEN SUPPLIES \\ \& \& \\ F. E. Palmer, Inc. \\ Florists \\ Landscape Gardeners \\ BROOKLINE, MASS.
}

220 Washington Street Telephone Regent 2300
Newton and Clyde Streets Telephone Regent 2350-M 


\section{Flowering Annuals}

\section{Plants and vines for window boxes and urns}

These plants are grown in pots and may be safely transplanted at any time during the summer.

Prices are for plants out of pots.

Each Per doz.

Ageratum, double, blue...............

$\$ 2.00$

Alyssum, Little Gem, white...

1.502 .00

Alternanthera, brilliant edging plant.......

Antirrhinum (Snapdragon) four colors....

Aster, early and late varieties (from frames)

$2.50-3.00$

white, pink, lavender, purple (per 100,\$3)

Begonia, Chatelaine, everblooming. ...50 and .75

Calendula, yellow, orange, gold............

Canna, large-flowering dwarf, in variety.... . .50

$2.00-3.00$

Centaurea cyanus (Bachelor Button) blue. .

5.00

Centaurea imperialis (Giant Sweet Sultan).

2.00

2.00

Coleus, colored foliage in several varieties.

Cosmos, early everblooming, pink, white...

2.00

2.00

Dracaena indivisa, for vases............ 2.00

Fuchsia, several varieties............50-1.00

Geranium, white, pink, red, scarlet....... . .35

Geranium, scented leaf kinds........... . .50

Gladiolus bulbs, named fancy varieties, assorted (per 100, $\$ 8.00) \ldots \ldots \ldots \ldots \ldots$.

Helichrysum (Everlasting) .............

Heliotrope ..................... . 50

Lemon verbena, sweet-scented foliage ..35 and .50

Lobelia, dwarf blue..................

Marigold, African, tall, orange and yellow .

Marigold, French, dwarf, orange and yellow

Nicotiana (Tobacco)

Affinis, white, fragrant, also pink hybrids New English Crimson ...............

Petunia, large fringed, fancy kinds ........

Petunia (Rosy Morn) single pink,

Single white and Blue ...............

Salpiglossis, mixed colors .....................

Salvia splendens (scarlet sage) ...........

Scabiosa (Mourning Bride, five colors .....

Stock (Gilliflower), in several colors ......

Swan River Daisy, azure blue

2.00

2.00

2.00

Verbena, pink, white, red, blue

2.00

2.50

3.00

3.00

2.00

2.50

2.00

2.00

4.00

2.00

Zinnia elegans, yellow, orange, flesh-pink, crimson, rose ....................

Giant Dahlia-flowered 


\section{Tender Trailing and Climbing Plants}

Cobaea scandens .................... $\quad .50 \quad \begin{array}{r}.50 \\ 5.00\end{array}$

English Ivy ..................50-1.00-3.00

Lobelia, trailing blue flower for boxes..... $\quad .20 \quad 2.00$

Senecia scandens (Summer Ivy) . . . . . . . . .25 3.00

Vinca major variegata (Trailing Periwinkle) $\quad .50 \quad 5.00$

\section{Vegetable Plants - from Pots Per doz.}

Egg Plants ............................ 1.00

Pepper, Bull Nose, Ruby King, sweet........... 1.00

Tomato, Bonny Best, Stone................. 1.00

Chives, Mint, clump, and Parsley, each .25

\section{Hardy Climbing Plants}

Ampelopsis veitchii (Boston Ivy).........

Ampelopsis-engelmanni (Woodbine) ......

Aristolochia sipho (Dutchman's Pipe) .....

Celastrus scandens (Bitter Sweet) ........

Clematis paniculata, white flowering......

Each

Euonymus radicans, hardy evergreen......

New Bronze Leaf variety........... $50-1.50-2.00$

Honeysuckle, Hall's everblooming........ $\quad .50-.75$

Roses, different sizes............... $\quad 1.50-2.00$
American Pillar
Gardenia
Baltimore Belle
Hiawatha
Dorothy Perkins
Paul's Scarlet Climber
Dr. Van Fleet
Roserie
Eugene Jacquette
Silver Moon
Excelsa
Tausendschon

Flower of Fairfield

Climbing Lady Ashtown, new pink hybrid tea

Mary Wallace, new bright pink Van Fleet

Star of Persia, new semi-dble, yellow....

2.00

3.00

Wistaria sinensis, blue................ 1.50

\section{Tested Flower and Vegetable Seeds}

Best Lawn Grass Seed, per 1b. .75, 1/2 1b. .40

Orders for shipment of plants and supplies by express or parcel post to your summer home will be given our careful attention.

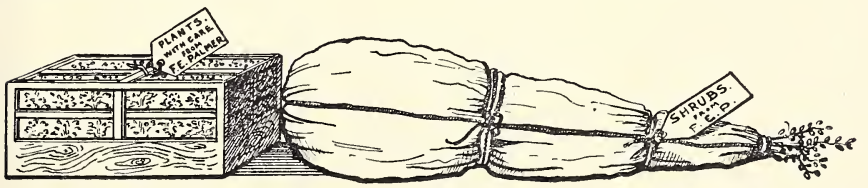


Achillea, white.................\$.35

Aconitum Fischeri (Blue Monkshood) . . . . . . .50

Alyssum saxatile (Gold Tuft), yellow..... . .50

Anemone japonica, pink and white..... ,50

Aquilegia (Columbine), mixed colors..... . .35

Asters, fall flowers, four choice varieties . . . $\quad .50$

Bellis peren nis (English Daisy) ..........

Buddleia (Butterfly Bush), lilac .......... .75

Campanula med (Canterbury Bell), pink, blue $\quad .35$

Campanula persicifolia.................. .35

Chrysanthemum, four colors........... .35

Coreopsis grandiflora, golden yellow ..... . .25

Delphinium, New English Hybrids...... . 1.00

Belladonna varieties .............. $\quad .50$

Dianthus barbalus (Sweet William) ...... .35

Dielytra spectabilis (Bleeding Heart) pink. 1.00

Digitalis (Foxglove) yellow and mixed.... .50

Gypsophila (Baby's Breath) double white. . .75

"Bristol Fairy," new variety .......... 1.50

Helenium, yellow, brown, red ......... .50

Hemerocallis thun, yellow............. .35

Hollyhocks, dble. yellow, pink, white, red. . .50

Iris germanica (German Iris) mixed ...... $\quad .50$

Iris kaempferi (Japanese Iris) . . . . . . . . . . . . . .

Iris pallida dalmatica................ . .50

Lily -of-the-Valley, large clumps . . . . . . . . $\quad .50$

Lupinus, blue, pink, white ............ .25

Lychnis viscaria, scarlet ............... .25

Myosotis (Forget-me-not)............. . . 25

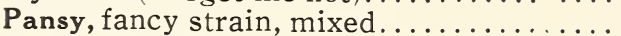

Peonies, Choice Named Varieties in pink, rose, red shades..............2.00-7.00

Collection of Choice Unnamed Kinds in different colors, large roots . . . . . . . 1.50

Festiva Maxima, large white........... 1.50

Officinalis rubra, earliest red.......... 2.00 Phlox, eight named varieties, tall growing. $\quad .50$

Phlox subulata, pink, white, clumps....75-1.00 Pyrethrum roseum, single mixed........ . . . .55 Rudbeckia (Golden Glow) yellow, clumps.. $\quad .50$ Sedum spectabile, pink, fall flowers....... . . .50 Veronica subsessilis, blue............. .35 